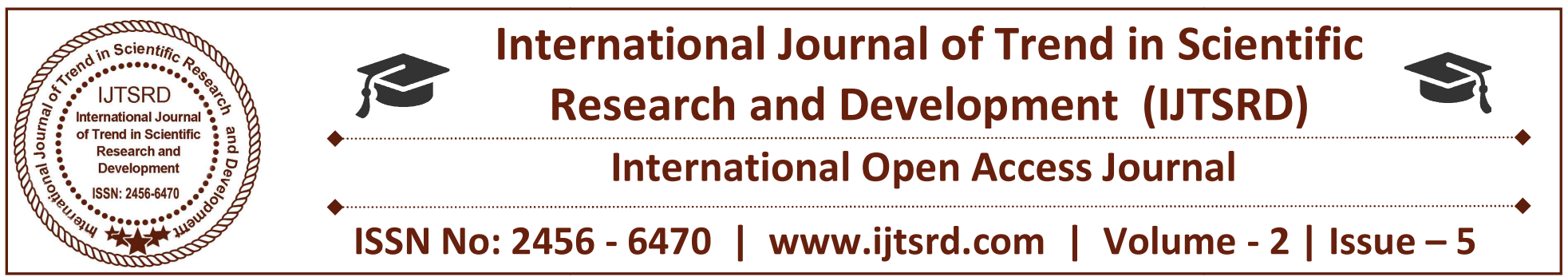

\title{
Reliability Modeling and Analysis of a Parallel Unit System with Priority to Repair over Replacement Subject to Maximum Operation and Repair Times
}

\author{
Reetu Rathee ${ }^{1}$, D. Pawar ${ }^{1}$, S. C. Malik ${ }^{2}$ \\ ${ }^{1}$ Assistant Professor, ${ }^{2}$ Professor \\ ${ }^{1}$ Amity Institute of Applied Science, AMITY University, Noida, Uttar Pradesh, India \\ ${ }^{2}$ Department of Statistics, M. D. University, Rohtak, Haryana, India
}

\begin{abstract}
This paper proposes the study of modelling and analysis of a two unit parallel system. A constant failure rate is considered for the units which are identical in nature. All repair activities like repair, replacement, preventive maintenance are mended immediately by a single server. The repair of the unit is done after its failure and if the fault is not rectified by the server within a given repair time, called maximum repair time, the unit replaced by new one. And, if there is no fault occurs up to a pre-fixed operation time, called maximum operation time, the unit undergoes for the preventive maintenance. The unit works as new after all repair activities done by the server. Priority to repair of one unit is given over the replacement of the other one. All random variables are statistically independent. The distribution for the failure, preventive maintenance and replacement rates are negative exponential whereas the distribution for all repair activities are taken as arbitrary with different probability density functions. Semi-Markov and regenerative point techniques are used to derive some reliability measures in steady state. The variation of MTSF, availability and profit function has been observed graphically for various parameters and costs.
\end{abstract}

Keywords: Parallel system, Preventive Maintenance, Replacement, Priority, Reliability Measures

\section{INTRODUCTION}

The general purpose of the modern world is to achieve the require performance level using the lowest possible cost. And, the parallel system works not only for maximize the profit but also for minimize the failure risk as well as cost. Keeping in view of their practical applications, reliability models of parallel systems have been developed and analyzed stochastically by the researchers and reliability engineers. Kishan and Kumar (2009) evaluated stochastically a parallel system using preventive maintenance. Further, kumar et al. (2010) and Malik and Gitanjali (2012) have analyzed cost-benefit of a parallel system subject to degradation after repair and arrival time of the server respectively. However, to enhance the profit of the system Reetu and Malik (2013) and Rathee and Chander (2014) developed parallel systems using the concept of priority.

Also, the objective of the present paper is to determine the reliability measures by giving the priority to one repair activity over the other ones. A constant failure rate is considered for the units which are identical in nature. All repair activities are done immediately by a single server. The repair of the unit is conducted after its failure and if the fault is not rectified by the server within a given repair time, the unit replaced by new one. And, if there is no fault occurs up to a pre-fixed operation time, the preventive maintenance is conducted. The unit works as new after all repair activities done by the server. Priority to repair of one unit is given over the replacement of the other one. All random variables are statistically independent. The distribution for the failure, preventive maintenance and replacement rates are 
negative exponential whereas the distribution for all repair activities are taken as arbitrary with different probability density functions. Semi-Markov and regenerative point techniques are used to derive some reliability measures in steady state. The variation of MTSF, availability and profit function has been observed graphically for various parameters and costs.

\section{NOTATIONS:}

\begin{tabular}{|c|c|}
\hline E & Set of regenerative states \\
\hline $\bar{E}$ & Set of non-regenerative states \\
\hline$\lambda$ & : Constant failure rate \\
\hline$\alpha_{0}$ & $\begin{array}{l}\text { The rate by which system undergoes } \\
\text { for preventive maintenance (called } \\
\text { maximum constant rate of operation } \\
\text { time) }\end{array}$ \\
\hline$\beta_{0}$ & $\begin{array}{l}\text { : The rate by which system underg } \\
\text { for replacement (called maxim } \\
\text { constant rate of repair time) }\end{array}$ \\
\hline
\end{tabular}

$\mathrm{FUr} / \mathrm{FWr}$ : The unit is failed and under repair/waiting for repair

FURp/FWRp : The unit is failed and under replacement/waiting for replacement

$\mathrm{UPm} / \mathrm{WPm}$ : The unit is under preventive maintenance/waiting for preventive maintenance

FUR/FWR : The unit is failed and under repair / waiting for repair continuously from previous state

FURP/FWRP : The unit is failed and under/waiting for replacement continuously from previous state

UPM/WPM : The unit is under/waiting for preventive maintenance continuously from previous state

$\mathrm{g}(\mathrm{t}) / \mathrm{G}(\mathrm{t}) \quad:$ pdf/cdf of repair time of the unit

$\mathrm{f}(\mathrm{t}) / \mathrm{F}(\mathrm{t}) \quad: \mathrm{pdf} / \mathrm{cdf}$ of preventive maintenance time of the unit

$\mathrm{r}(\mathrm{t}) / \mathrm{R}(\mathrm{t}) \quad:$ pdf/cdf of replacement time of the unit $\mathrm{q}_{\mathrm{ij}}(\mathrm{t}) / \mathrm{Q}_{\mathrm{ij}}(\mathrm{t})$ : pdf / cdf of passage time from regenerative state $\mathrm{Si}$ to a regenerative stateSjor to a failed state $\mathrm{Sj}$ without visiting any other regenerative state in $(0, \mathrm{t}]$

$\mathrm{q}_{\mathrm{ij}, k r}(\mathrm{t}) / \mathrm{Q}_{\mathrm{ij}, \mathrm{kr}}(\mathrm{t})$ : pdf/cdf of direct transition time from regenerative state $\mathrm{Si}$ to a regenerative state $\mathrm{Sj}$ or to a failed state $\mathrm{Sj}$ visiting state $\mathrm{Sk}, \mathrm{Sr}$ once in $(0, \mathrm{t}]$

$\mathrm{M}_{\mathrm{i}}(\mathrm{t}) \quad$ : Probability that the system up initially in state $S_{i} \in E$ is up at time $\mathrm{t}$ without visiting to any regenerative state

$\mathrm{W}_{\mathrm{i}}(\mathrm{t}) \quad$ : Probability that the server is busy in the state $S_{i}$ up to time ' $t$ ' without making any transition to any other regenerative state or returning to the same state via one or more nonregenerative states.

: The mean sojourn time in state $S_{i}$ which is given by

$$
\mu_{i}=E(T)=\int_{0}^{\infty} P(T>t) d t=\sum_{j} m_{i j},
$$

where $T$ denotes the time to system failure.

$\mathrm{m}_{\mathrm{ij}}$ :Contribution to mean sojourn time $\left(\mu_{\mathrm{i}}\right)$ in state $\mathrm{S}_{\mathrm{i}}$ when system transits

$$
\text { directlyto state } \mathrm{S}_{\mathrm{j}} \mathrm{So} \text { that } \mu_{i}=\sum_{j} m_{i j} \text { and } \mathrm{m}_{\mathrm{ij}}=
$$

$$
\int t d Q_{i j}(t)=-q_{i j}^{*}(0)
$$

\&/(C) : Symbol for Laplace-Stieltjes convolution/Laplace convolution

Symbol for Laplace Transformation /LaplaceStieltjes Transformation

The states $\mathrm{S}_{0}, \mathrm{~S}_{1}, \mathrm{~S}_{2}, \mathrm{~S}_{4}, \mathrm{~S}_{6}$ and $\mathrm{S}_{7}$ are regenerative while the states $S_{3}, S_{5}, S_{8}, S_{9}, S_{10}, S_{11}$ and $S_{12}$ are nonregenerative as shown in figure 1 . 
International Journal of Trend in Scientific Research and Development (IJTSRD) ISSN: 2456-6470

TRANSITION STATE DIAGRAM

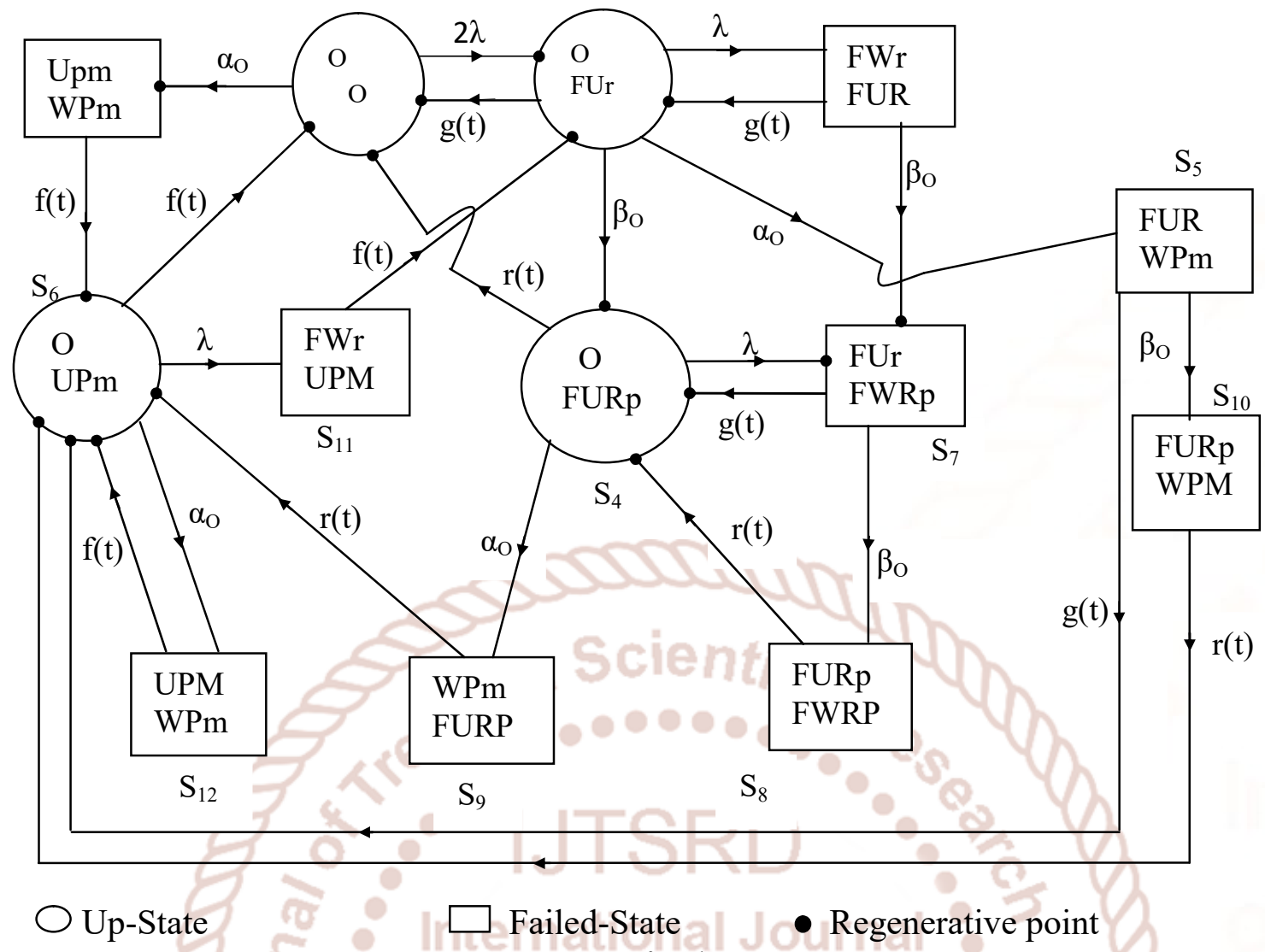

Fig. 1

\section{Transition Probabilities and Mean Sojourn Times}

Simple probabilistic considerations yield the following expressions for the non-zero elements

$p_{i j}=Q_{i j}(\infty)=\int_{0}^{\infty} q_{i j}(t) d t$ as

$p_{01}=\frac{2 \lambda}{2 \lambda+\alpha_{0}}, p_{02}=\frac{\alpha_{0}}{2 \lambda+\alpha_{0}}, p_{15}=\frac{\alpha_{0}}{\left(\lambda+\alpha_{0}+\beta_{0}\right)}\left(1-g^{*}\left(\lambda+\alpha_{0}+\beta_{0}\right)\right)$,

$p_{40}=r^{*}\left(\lambda+\alpha_{0}\right), p_{13}=\frac{\lambda}{\left(\lambda+\alpha_{0}+\beta_{0}\right)}\left(1-g^{*}\left(\lambda+\alpha_{0}+\beta_{0}\right)\right), p_{60}=f^{*}\left(\lambda+\alpha_{0}\right)$,

$p_{10}=g^{*}\left(\lambda+\alpha_{0}+\beta_{0}\right), p_{17.3}=\frac{\lambda}{\left(\lambda+\alpha_{0}+\beta_{0}\right)}\left(1-g^{*}\left(\beta_{0}\right)\right)\left(1-g^{*}\left(\lambda+\alpha_{0}+\beta_{0}\right)\right)$,

$p_{6,12}=p_{66.12}=\frac{\alpha_{0}}{\left(\lambda+\alpha_{0}\right)}\left(1-f^{*}\left(\lambda+\alpha_{0}\right)\right), p_{6,11}=p_{61.11}=\frac{\lambda}{\left(\lambda+\alpha_{0}\right)}\left(1-f^{*}\left(\lambda+\alpha_{0}\right)\right)$,

$p_{49}=p_{46.9}=\frac{\alpha_{0}}{\left(\lambda+\alpha_{0}\right)}\left(1-r^{*}\left(\lambda+\alpha_{0}\right)\right), p_{37}=p_{5,10}=p_{78}=p_{74.8}=1-g^{*}\left(\beta_{0}\right)$,

$p_{14}=\frac{\beta_{0}}{\left(\lambda+\alpha_{0}+\beta_{0}\right)}\left(1-g^{*}\left(\lambda+\alpha_{0}+\beta_{0}\right)\right), p_{47}=\frac{\lambda}{\left(\lambda+\alpha_{0}\right)}\left(1-r^{*}\left(\lambda+\alpha_{0}\right)\right)$,

$p_{31}=p_{56}=p_{74}=g^{*}\left(\beta_{0}\right), p_{26}=p_{84}=p_{96}=p_{10,6}=p_{11,1}=p_{12,6}=1$

\section{It can be easily verify that}

$p_{01}+p_{02}=p_{10}+p_{13}+p_{14}+p_{15}=p_{26}=p_{40}+p_{47}+p_{49}=p_{60}+p_{6,11}+p_{6,12}=1$

$p_{10}+p_{14}+p_{11.3}+p_{16.5}+p_{16.5,10}+p_{17.3}=p_{40}+p_{47}+p_{46.9}=1$

$p_{60}+p_{61.11}+p_{66.12}=p_{74}+p_{74.8}=1$ 
The mean sojourn times $\left(\mu_{i}\right)$ is in the state $S_{\mathbf{i}}$ are

$\mu_{0}=m_{01}+m_{02}, \mu_{1}=m_{10}+m_{13}+m_{14}+m_{15}, \mu_{2}=m_{26}, \mu_{4}=m_{40}+m_{47}+m_{49}, \mu_{6}=m_{60}+m_{6,11}+m_{6,12}$,

$\mu_{1}^{\prime}=m_{10}+m_{14}+m_{11.3}+m_{16.5}+m_{16.5,10}+m_{17.3}$,

$\mu_{4}^{\prime}=m_{40}+m_{47}+m_{46.9}, \mu_{7}=m_{74}+m_{74.8}$,

\section{Reliability and Mean Time to System Failure (MTSF)}

Let $\phi_{\mathrm{i}}(\mathrm{t})$ be the cdf of first passage time from regenerative state $\mathrm{Si}$ to a failed state. Regarding thefailed state as absorbing state, we have the following recursive relations for $\phi_{\mathrm{i}}(\mathrm{t})$ :

$\phi_{0}(t)=Q_{01}(t) \& \phi_{1}(t)+Q_{02}(t)$

$\phi_{1}(t)=Q_{10}(t) \& \phi_{0}(t)+Q_{14}(t) \& \phi_{4}(t)+Q_{13}(t)+Q_{15}(t)$

$\phi_{4}(t)=Q_{40}(t) \& \phi_{0}(t)+Q_{48}(t)+Q_{49}(t)$

Taking LST of above relation (7.4) and solving for $\Phi_{0}^{* *}(\mathrm{~s})$, we have

$R^{*}(s)=\frac{1-\phi^{* *}(s)}{s}$

The reliability of the system model can be obtained by taking Inverse Laplace transform of (3). The mean time to system failure (MTSF) is given by

$\operatorname{MTSF}=\lim _{s \rightarrow 0} \frac{1-\phi^{* *}(s)}{s}=\frac{N}{D}$

Where

$N=\mu_{0}+p_{01} \mu_{1}+p_{01} p_{14} \mu_{4}$ and $D=1-p_{01} p_{10}-p_{01} p_{14} p_{40}$

\section{Steady State Availability}

Let $\mathrm{A}_{\mathrm{i}}(\mathrm{t})$ be the probability that the system is in up-state at instant ' $\mathrm{t}$ ' given that thesystementered regenerative state $\mathrm{Si}$ at $\mathrm{t}=0$.The recursive relations for $A_{i}(t)$ are given as:

$$
\begin{aligned}
& A_{0}(t)=M_{0}(t)+q_{01}(t) \odot A_{1}(t)+q_{02}(t) \odot A_{2}(t) \\
& A_{1}(t)=M_{1}(t)+q_{10}(t) \odot A_{0}(t)+q_{11.3}(t) \odot A_{1}(t)+q_{14}(t) \odot A_{4}(t) \\
& +q_{17.3}(t) \odot A_{7}(t)+\left(q_{16.5}(t)+q_{16.5,10}(t)\right) \odot A_{6}(t) \\
& A_{2}(t)=q_{26}(t) \subseteq A_{6}(t) \\
& A_{4}(t)=M_{4}(t)+q_{40}(t) \odot A_{0}(t)+q_{47}(t) \odot A_{7}(t)+q_{46.9}(t) \odot A_{6}(t) \\
& A_{6}(t)=M_{6}(t)+q_{60}(t) \odot A_{0}(t)+q_{61.11}(t) \odot A_{1}(t)+q_{66.12}(t) \odot A_{6}(t) \\
& A_{7}(t)=\left(q_{74}(t)+q_{74.8}(t)\right) @ A_{4}(t)
\end{aligned}
$$

Where

$$
\begin{aligned}
& M_{0}(t)=e^{-\left(2 \lambda+\alpha_{0}\right) t}, M_{1}(t)=e^{-\left(\lambda+\alpha_{0}+\beta_{0}\right) t} \overline{G(t)}, \\
& M_{4}(t)=e^{-\left(\lambda+\alpha_{0}\right) t} \overline{R(t)}, M_{6}(t)=e^{-\left(\lambda+\alpha_{0}\right) t} \overline{F(t)}
\end{aligned}
$$

Taking LT of above relation (6) and solving for $\mathrm{A}_{0} *(\mathrm{~s})$. The steady state availability is given by

$$
A_{0}(\infty)=\lim _{s \rightarrow 0} s A_{0}^{*}(s)=\frac{N_{1}}{D_{1}}
$$

Where

$$
\begin{aligned}
N_{1}= & \mu_{0}\left[\left(1-p_{47}\right)\left\{p_{60}\left(1-p_{11.3}\right)+p_{61.11} p_{10}\right\}+p_{61.11} p_{40}\left(p_{14}+p_{17.3}\right)\right] \\
& +\mu_{6}\left[\left(1-p_{47}\right)\left\{p_{01}\left(p_{16.5}+p_{16.5,10}\right)+p_{02}\left(1-p_{11.3}\right)\right\}\right. \\
& \left.+p_{01} p_{46.9}\left(p_{14}+p_{17.3}\right)\right]+\mu_{1}\left(1-p_{47}\right)\left\{p_{01}\left(1-p_{66.12}\right)-p_{02} p_{61.11}\right\} \\
& +\mu_{4}\left(p_{14}+p_{17.3}\right)\left\{p_{01} p_{60}+p_{61.11}\right\}
\end{aligned}
$$


International Journal of Trend in Scientific Research and Development (IJTSRD) ISSN: 2456-6470

$$
\begin{aligned}
D_{1}= & \left(\mu_{0}+\mu_{2} p_{02}\right)\left[\left(1-p_{47}\right)\left\{p_{60}\left(1-p_{11.3}\right)+p_{61.11} p_{10}\right\}+p_{61.11} p_{40}\left(p_{14}+p_{17.3}\right)\right] \\
& +\mu_{6}^{\prime}\left[\left(1-p_{47}\right)\left\{p_{01}\left(p_{16.5}+p_{16.5,10}\right)+p_{02}\left(1-p_{11.3}\right)\right\}+p_{01} p_{46.9}\left(p_{14}+p_{17.3}\right)\right] \\
& +\left(p_{01} p_{60}+p_{61.11}\right)\left\{\mu_{4}^{\prime}\left(p_{14}+p_{17.3}\right)+\mu_{7}^{\prime}\left(p_{14} p_{47}+p_{17.3}\right)\right\} \\
& +\mu_{1}^{\prime}\left(1-p_{47}\right)\left\{p_{01}\left(1-p_{66.12}\right)-p_{02} p_{61.11}\right\}
\end{aligned}
$$

\section{Busy Period Analysis for Server}

\section{A. Due to Repair}

Let $B_{i}^{R}(t)$ be the probability that the server is busy in repairof the unit at an instant' $\mathrm{t}$ ' given that the system entered regenerative state $\mathrm{Si}$ at $\mathrm{t}=0$. The recursive relations for $B_{i}^{R}(t)$ are as follows:

$$
\begin{aligned}
& B_{0}^{R}(t)=q_{01}(t) \Subset B_{1}^{R}(t)+q_{02}(t) \Subset B_{2}^{R}(t) \\
& B_{1}^{R}(t)=W_{1}(t)+q_{10}(t) \subseteq B_{0}^{R}(t)+q_{11.3}(t) \subseteq B_{1}^{R}(t)+q_{14}(t) \subseteq B_{4}^{R}(t) \\
& +q_{17.3}(t) @ B_{7}^{R}(t)+\left(q_{16.5}(t)+q_{16.5,10}(t)\right) \subseteq B_{6}^{R}(t) \\
& B_{2}^{R}(t)=q_{26}(t) @ B_{6}^{R}(t) \\
& B_{4}^{R}(t)=q_{40}(t) \subseteq B_{0}^{R}(t)+q_{47}(t) \subseteq B_{7}^{R}(t)+q_{46.9}(t) \subseteq B_{6}^{R}(t) \\
& B_{6}^{R}(t)=q_{60}(t) \odot B_{0}^{R}(t)+q_{61.11}(t) \odot B_{1}^{R}(t)+q_{66.12}(t) \subseteq B_{6}^{R}(t) \\
& B_{7}^{R}(t)=W_{7}(t)+\left(q_{74}(t)+q_{74.8}(t)\right) \subset B_{4}^{R}(t)
\end{aligned}
$$

Where,

$W_{1}(t)=e^{-\left(\lambda+\alpha_{0}+\beta_{0}\right) t} \overline{G(t)}+\left(\lambda e^{-\left(\lambda+\alpha_{0}+\beta_{0}\right) t}(1) \overline{G(t)}+\left(\alpha_{0} e^{-\left(\lambda+\alpha_{0}+\beta_{0}\right) t}(1) \overline{G(t)}\right.\right.$

and $W_{7}(t)=e^{-\beta_{0} t} \overline{G(t)}$

Taking LT of above relation (11) and solving for $B_{0}^{R^{*}}(s)$. The time for which serveris busydue to repair is given by

$$
B_{0}^{R}(\infty)=\lim _{s \rightarrow 0} s B_{0}^{R^{*}}(s)=\frac{N_{2}}{D_{1}}
$$

Where,

$$
N_{2}=W_{1}^{*}(0)\left(1-p_{47}\right)\left\{p_{01}\left(1-p_{66.12}\right)-p_{02} p_{61.11}\right\}+W_{7}^{*}(0)\left(p_{14} p_{47}+p_{17.3}\right)\left\{p_{01} p_{60}+p_{61.11}\right\}
$$

and $\mathrm{D}_{1}$ is already mentioned.

\section{B. Due to Replacement}

Let $B_{i}^{R p}(t)$ be the probability that the server is busy in replacement of the unit at an instant ' $\mathrm{t}$ ' given that the system entered regenerative state $\mathrm{Si}$ at $\mathrm{t}=0$. The recursive relations for $B_{i}^{R p}(t)$ are as follows:

$$
\begin{aligned}
& B_{0}^{R p}(t)=q_{01}(t) @ B_{1}^{R p}(t)+q_{02}(t) @ B_{2}^{R p}(t) \\
& B_{1}^{R p}(t)=q_{10}(t) \Subset B_{0}^{R p}(t)+q_{11.3}(t) \Subset B_{1}^{R p}(t)+q_{14}(t) \Subset B_{4}^{R p}(t) \\
& +q_{17.3}(t) @ B_{7}^{R p}(t)+\left(q_{16.5}(t)+q_{16.5,10}(t)\right) @ B_{6}^{R p}(t) \\
& B_{2}^{R p}(t)=q_{26}(t) \subset B_{6}^{R p}(t) \\
& B_{4}^{R p}(t)=W_{4}(t)+q_{40}(t) \Subset B_{0}^{R p}(t)+q_{47}(t) \Subset B_{7}^{R p}(t)+q_{46.9}(t) \Subset B_{6}^{R p}(t) \\
& B_{6}^{R p}(t)=q_{60}(t) \Subset B_{0}^{R p}(t)+q_{61.11}(t) \Subset B_{1}^{R p}(t)+q_{66.12}(t) \Subset B_{6}^{R p}(t) \\
& B_{7}^{R p}(t)=\left(q_{74}(t)+q_{74.8}(t)\right) \subset B_{4}^{R p}(t)
\end{aligned}
$$

Where, 
$W_{4}(t)=e^{-\left(\lambda+\alpha_{0}\right) t} \overline{R(t)}+\left(\alpha_{0} e^{-\left(\lambda+\alpha_{0}\right) t} \subseteq 1\right) \overline{R(t)}$

Taking LT of above relation (15) and solving for $B_{0}^{R p^{*}}(s)$. The time for which server is busy due to replacement is given by

$$
B_{0}^{R p}(\infty)=\lim _{s \rightarrow 0} s B_{0}^{R p^{*}}(s)=\frac{N_{3}}{D_{1}}
$$

Where,

$$
N_{3}=W_{4}^{*}(0)\left(p_{14}+p_{17.3}\right)\left(p_{01} p_{60}+p_{61.11}\right) \text { and } \mathrm{D}_{1} \text { is already mentioned. }
$$

\section{Due to Preventive Maintenance}

Let $B_{i}^{P}(t)$ be the probability that the server is busy in preventive maintenance of the unit at an instant' $\mathrm{t}$ ' given that the system entered regenerative state $\mathrm{Si}$ at $\mathrm{t}=0$. The recursive relations for $B_{i}^{P}(t)$ are as follows:

$$
\begin{aligned}
& B_{0}^{P}(t)=q_{01}(t) \Subset B_{1}^{P}(t)+q_{02}(t) \Subset B_{2}^{P}(t) \\
& B_{1}^{P}(t)=q_{10}(t) \Subset B_{0}^{P}(t)+q_{11.3}(t) \Subset B_{1}^{P}(t)+q_{14}(t) \Subset B_{4}^{P}(t) \\
& +q_{17.3}(t) \subseteq B_{7}^{P}(t)+\left(q_{16.5}(t)+q_{16.5,10}(t)\right) \subseteq B_{6}^{P}(t) \\
& B_{2}^{P}(t)=W_{2}(t)+q_{26}(t) \subseteq B_{6}^{P}(t) \\
& B_{4}^{P}(t)=q_{40}(t) \Subset B_{0}^{P}(t)+q_{47}(t) \subseteq B_{7}^{P}(t)+q_{46.9}(t) @ B_{6}^{P}(t) \\
& B_{6}^{P}(t)=W_{6}(t)+q_{60}(t) \Subset B_{0}^{P}(t)+q_{61.11}(t) \subseteq B_{1}^{P}(t)+q_{66.12}(t) \Subset B_{6}^{P}(t) \\
& B_{7}^{P}(t)=\left(q_{74}(t)+q_{74.8}(t)\right)\left(C B_{4}^{P}(t)\right.
\end{aligned}
$$

Where,

$$
W_{6}(t)=e^{-\left(\lambda+\alpha_{0}\right) t} \overline{F(t)}+\left(\alpha_{0} e^{-\left(\lambda+\alpha_{0}\right) t} @ 1\right) \overline{F(t)}+\left(\lambda e^{-\left(\lambda+\alpha_{0}\right) t} @ 1\right) \overline{F(t)} \text { and } W_{2}(t)=\overline{F(t)}
$$

Taking LT of above relation (19) and solving for $B_{0}^{P^{*}}(s)$. The time for which server is busy due to preventive maintenance is given by

$$
B_{0}^{P}(\infty)=\lim _{s \rightarrow 0} s B_{0}^{P^{*}}(s)=\frac{N_{4}}{D_{1}}
$$

Where,

$$
\begin{aligned}
N_{4}= & W_{2}^{*}(0) p_{02}\left[\left(1-p_{47}\right)\left\{p_{60}\left(1-p_{11.3}\right)+p_{61.11} p_{10}\right\} p_{61.11} p_{40}\left(p_{14}+p_{17.3}\right)\right] \\
& +W_{6}^{*}(0)\left[\left(1-p_{47}\right)\left\{p_{01}\left(p_{16.5}+p_{16.5,10}\right)+p_{02}\left(1-p_{11.3}\right)\right\}+p_{01} p_{46.9}\left(p_{14}+p_{17.3}\right)\right]
\end{aligned}
$$

and $\mathrm{D}_{1}$ is already mentioned.

\section{Expected Number of Repairs}

Let $R_{i}(t)$ be the expected number of repairs by the server in $(0, \mathrm{t}]$ given that the system entered the regenerative state $\mathrm{Si}$ at $\mathrm{t}=0$. The recursive relations for $R_{i}(t)$ are given as:

$$
\begin{aligned}
R_{0}(t)= & Q_{01}(t) \& R_{1}(t)+Q_{02}(t) \& R_{2}(t) \\
R_{1}(t)= & Q_{10}(t) \&\left[1+R_{0}(t)\right]+Q_{11.3}(t) \&\left[1+R_{1}(t)\right]+Q_{14}(t) \& R_{4}(t) \\
& +Q_{17.3}(t) \& R_{7}(t)+Q_{16.5}(t) \&\left[1+R_{6}(t)\right]+Q_{16.5,10}(t) \& R_{6}(t) \\
R_{2}(t)= & Q_{26}(t) \& R_{6}(t) \\
R_{4}(t)= & Q_{40}(t) \& R_{0}(t)+Q_{47}(t) \& R_{7}(t)+Q_{46.9}(t) \& R_{6}(t) \\
R_{6}(t)= & Q_{60}(t) \& R_{0}(t)+Q_{61.11}(t) \& R_{1}(t)+Q_{66.12}(t) \& R_{6}(t) \\
R_{7}(t)= & Q_{74}(t) \&\left[1+R_{4}(t)\right]+Q_{74.8}(t) \& R_{4}(t)
\end{aligned}
$$


Taking LST of above relations (23) and solving for $R_{0}^{* *}(s)$. The expected no. of repairs per unit time by the server are giving by

$$
R_{0}(\infty)=\lim _{s \rightarrow 0} s R_{0}^{* *}(s)=\frac{N_{5}}{D_{1}}
$$

Where,

$$
\begin{aligned}
N_{5}= & \left(p_{10}+p_{11.3}+p_{16.5}\right)\left(1-p_{47}\right)\left\{p_{01}\left(1-p_{66.12}\right)-p_{02} p_{61.11}\right\} \\
& +p_{74}\left(p_{14} p_{47}+p_{17.3}\right)\left(p_{01} p_{60}+p_{61.11}\right)
\end{aligned}
$$

and $\mathrm{D}_{1}$ is already mentioned.

\section{Expected Number of Replacements}

Let $R p_{i}(t)$ be the expected number of replacements by the server in $(0, \mathrm{t}]$ given that the system entered the regenerative state $\mathrm{Si}$ at $\mathrm{t}=0$. The recursive relations for $R p_{i}(t)$ are given as:

$$
\begin{aligned}
R p_{0}(t) & =Q_{01}(t) \& R p_{1}(t)+Q_{02}(t) \& R p_{2}(t) \\
R p_{1}(t)= & Q_{10}(t) \& R p_{0}(t)+Q_{11.3}(t) \& R p_{1}(t)+Q_{14}(t) \& R p_{4}(t) \\
& +Q_{17.3}(t) \& R p_{7}(t)+Q_{16.5,10}(t) \&\left[1+R p_{6}(t)\right]+Q_{16.5}(t) \& R p_{6}(t) \\
R p_{2}(t) & =Q_{26}(t) \& R p_{6}(t) \\
R p_{4}(t)= & Q_{40}(t) \&\left[1+R p_{0}(t)\right]+Q_{47}(t) \& R p_{7}(t)+Q_{46.9}(t) \&\left[1+R p_{6}(t)\right] \\
R p_{6}(t)= & Q_{60}(t) \& R p_{0}(t)+Q_{61.11}(t) \& R p_{1}(t)+Q_{66.12}(t) \& R p_{6}(t) \\
R p_{7}(t)= & \left.Q_{74}(t) \& R p_{4}(t)\right]+Q_{74.8}(t) \&\left[1+R p_{4}(t)\right]
\end{aligned}
$$

Taking LST of above relations (26) and solving for $R p_{0}^{* *}(s)$. The expected no. of replacements per unit time by the server are giving by

$$
R p_{0}(\infty)=\lim _{s \rightarrow 0} s p_{0}^{* *}(s)=\frac{N_{6}}{D_{1}}
$$

Where,

$$
\begin{aligned}
N_{6}= & p_{16.5,10}\left(1-p_{47}\right)\left\{p_{01}\left(1-p_{66.12}\right)-p_{02} p_{61.11}\right\} \\
& +\left(p_{40}+p 46.9 p\right)\left(p_{14}+p_{17.3}\right)\left\{p_{01} p_{60}+p_{61.11}\right\} \\
& +p_{74.8}\left(p_{14} p_{47}+p_{17.3}\right)\left(p_{01} p_{60}+p_{61.11}\right)
\end{aligned}
$$

and $\mathrm{D}_{1}$ is already mentioned.

\section{Expected Number of Preventive Maintenances}

Let $P_{i}(t)$ be the expected number of preventive maintenance by the server in $(0, \mathrm{t}]$ given that the system entered the regenerative state $\mathrm{S}_{\mathrm{i}}$ at $\mathrm{t}=0$. The recursive relations for $P_{i}(t)$ are given as:

$$
\begin{aligned}
P_{0}(t)= & Q_{01}(t) \& P_{1}(t)+Q_{02}(t) \& P_{2}(t) \\
P_{1}(t)= & Q_{10}(t) \& P_{0}(t)+Q_{11.3}(t) \& P_{1}(t)+Q_{14}(t) \& P_{4}(t) \\
& +Q_{17.3}(t) \& P_{7}(t)+\left\{Q_{16.5}(t)+Q_{16.5,10}(t)\right\} \& P_{6}(t) \\
P_{2}(t)= & Q_{26}(t) \&\left[1+P_{6}(t)\right] \\
P_{4}(t)= & Q_{40}(t) \& P_{0}(t)+Q_{47}(t) \& P_{7}(t)+Q_{46.9}(t) \& P_{6}(t) \\
P_{6}(t)= & Q_{60}(t) \&\left[1+P_{0}(t)\right]+Q_{61.11}(t) \&\left[1+P_{1}(t)\right]+Q_{66.12}(t) \&\left[1+P_{6}(t)\right] \\
P_{7}(t)= & \left\{Q_{74}(t)+Q_{74.8}(t)\right\} \& P_{4}(t)
\end{aligned}
$$

Taking LST of above relations (29) and solving for $P_{0}^{* *}(s)$. The expected no. of preventive maintenances per unit time by the server are giving by 
$P_{0}(\infty)=\lim _{s \rightarrow 0} s P_{0}^{* *}(s)=\frac{N_{7}}{D_{1}}$

Where,

$$
\begin{aligned}
N_{7}= & p_{02}\left[\left(1-p_{47}\right)\left\{p_{60}\left(1-p_{11.3}\right)+p_{61.11} p_{10}\right\}+p_{61.11} p_{40}\left(p_{14}+p_{17.3}\right)\right] \\
& +\left[\left(1-p_{47}\right)\left\{p_{01}\left(p_{16.5}+p_{16.5,10}\right)+p_{02}\left(1-p_{11.3}\right)\right\}+p_{01} p_{46.9}\left(p_{14}+p_{17.3}\right)\right]
\end{aligned}
$$

and $\mathrm{D}_{1}$ is already mentioned.

\section{Profit Analysis}

The profit incurred to the system model in steady state can be obtained as

$$
P=K_{0} A_{0}-K_{1} B_{0}^{R}-K_{2} B_{0}^{R p}-K_{3} B_{0}^{P}-K_{4} R_{1}-K_{5} R p_{0}-K_{6} P_{0}
$$

Where,

$P=$ Profit of the system model after reducing cost per unit time busy of the server and

cost per repair activity per unit time

$K_{0}=$ Revenue per unit up-time of the system

$K_{1}=$ Cost per unit time for which server is busy due to repair

$K_{2}=$ Cost per unit time for which server is busy due to replacement

$K_{3}=$ Cost per unit time for which server is busy due to preventive maintenance

$K_{4}=$ Cost per repair per unit time

$K_{5}=$ Cost per replacement per unit time

$K_{6}=$ Cost per preventive maintenance per unit time

\section{CONCLUSION}

After solving the equations of MTSF, availability and profit function for the particular case $\mathrm{g}(\mathrm{t})=\theta e^{-\theta t}, \mathrm{r}(\mathrm{t})=$ $\beta e^{-\beta t}$ and $\mathrm{f}(\mathrm{t})=\alpha e^{-\alpha t}$, we conclude that

$>$ These reliability measures are increasing as the repair rate $\theta$, replacement rate $\beta$ increases while their values decline with the increase of failure rate $\lambda$ and the rate $\alpha_{0}$ by which the unit undergoes for preventive maintenance.

$>$ The MTSF and availability keep on upwards with the increase of the rate $\beta_{0}$ by which unit undergoes for replacement but profit decreases.

The system model becomes more profitable when we increase the preventive maintenance rate $\alpha$.

\section{GRAPHS FOR PARTICULAR CASE}

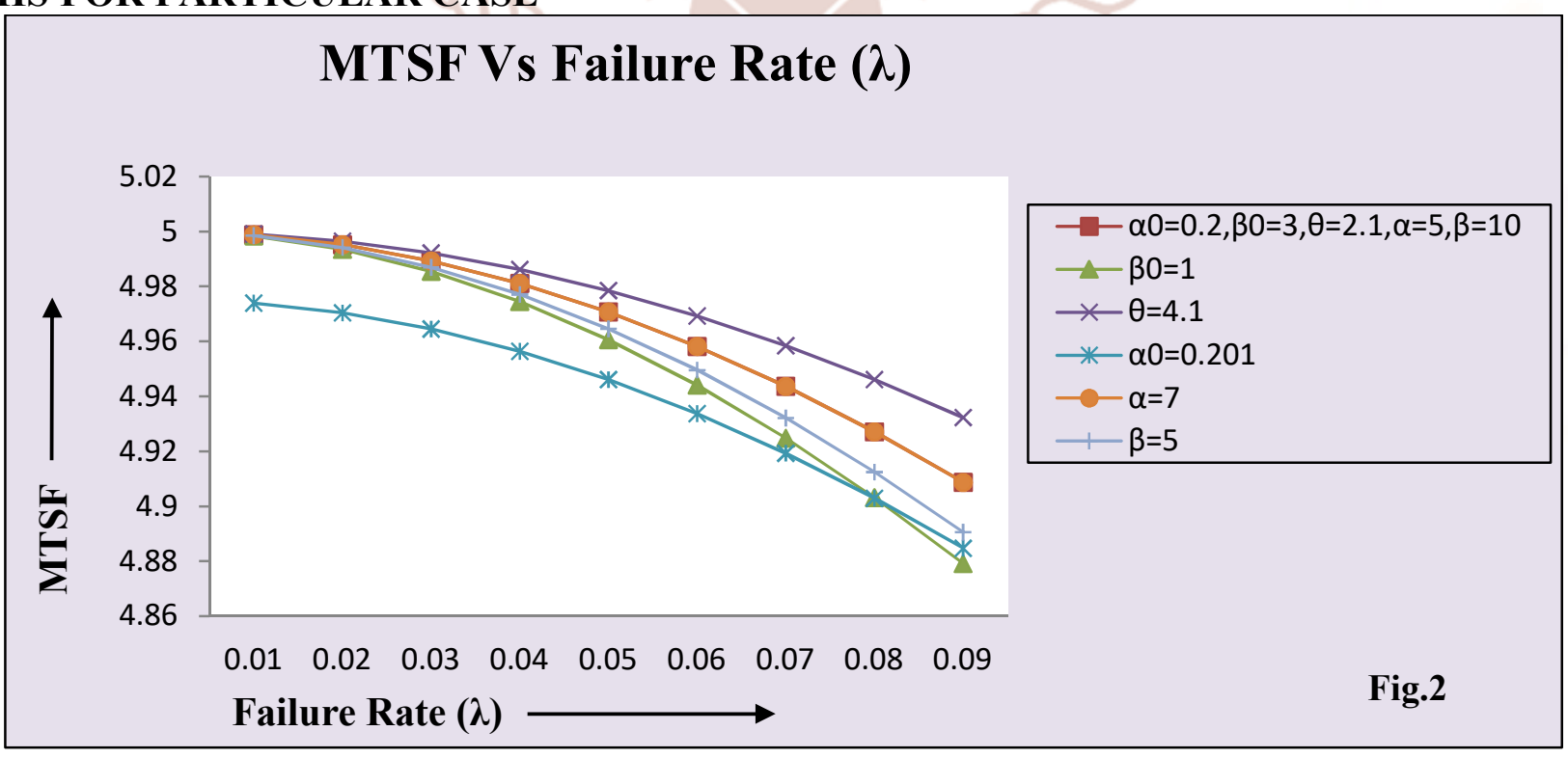



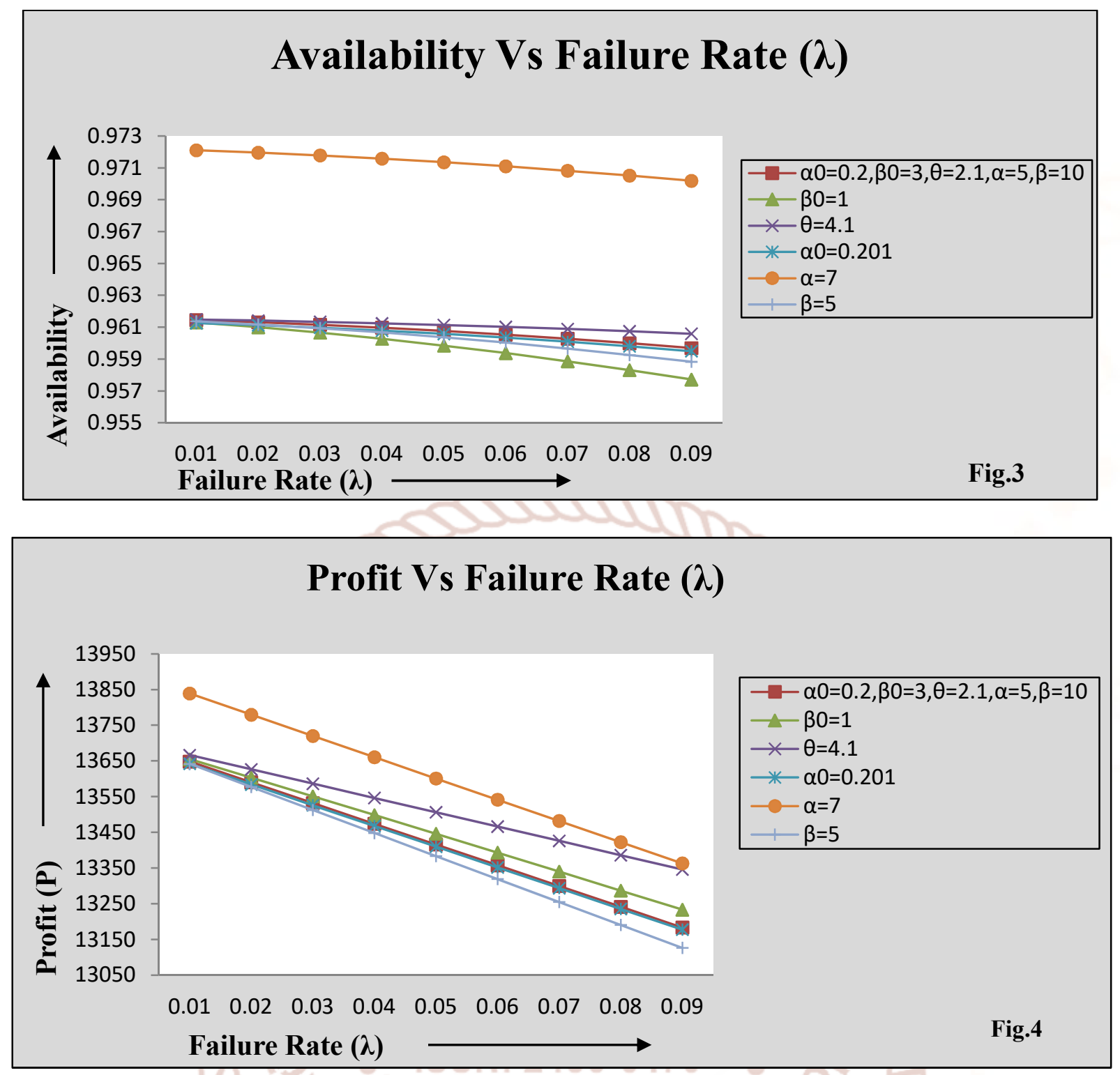

\section{REFERENCES}

1. R. Kishan and M. Kumar (2009), "Stochastic analysis of a two-unit parallel system with preventive maintenance", Journal of Reliability and Statistical Studies, vol. 22, pp. 31-38.

2. Kumar, Jitender, Kadyan, M.S. and Malik, S.C. (2010): Cost-benefit analysis of a two-unit parallel system subject to degradation after repair. Journal of Applied Mathematical Sciences, Vol.4 (56), pp.2749-2758.

3. Malik S.C. and Gitanjali (2012). Cost-Benefit Analysis of a Parallel System with Arrival Time of the Server and Maximum Repair Time. International Journal of Computer Applications, 46 (5): 39-44.
4. Reetu and Malik S.C. (2013),A Parallel System with Priority to Preventive Maintenance Subject to Maximum Operation and Repair Time. American journal of Mathematics and Statistics, Vol. 3(6), pp. 436-440.

5. Rathee R. and Chander S. (2014), A Parallel System with Priority to Repair over Preventive Maintenance Subject to Maximum Operation and Repair Time. International Journal of Statistics and Reliability Engineering, Vol. 1(1), pp. 57-68. 